\title{
Using health psychology to help patients: promoting healthy choices
}

Elizabeth Barley

Victoria Lawson

This article describes behaviour change techniques that nurses can use to help individual patients to make and stick to healthy choices. These include helping patients to set goals that are specific, measureable, achievable, relevant and timely (SMART), promoting self-monitoring and providing feedback and motivational interviewing. The process for delivering these techniques is described and the evidence for them discussed. Simply providing brief advice and follow up can lead to behaviour change, even in people who have not expressed a desire to change. The techniques are designed to be brief and feasible to use in routine practice. Using them can help nurses to apply the NHS policy of Making Every Contact Count so that their patients achieve long-term benefit.

In this article on using health psychology in nursing, the authors describe simple behaviour change techniques that nurses can use to help individual patients make and stick to healthy choices. Even if only a small proportion of each nurse's patients are helped in this way, there will be benefits at the population level in overall quality of life and reduced burden to the NHS. This reasoning is behind the NHS policy 'Making Every Contact Count' (MECC) (Bailey et al, 2012) and is supported by Royal College of Nursing (RCN) guidance that:

\section{'Every interaction in every location should be seen as an opportunity to promote health and prevent illness.'}

RCN, 2012: 24

In 2014, the National Institute for Health and Care Excellence (NICE) issued comprehensive guidance on the evidence for what helps individuals change their behaviour, focusing on behaviours relating to alcohol, diet, physical activity and smoking (NICE, 2014). The guidance highlights the value of 'very brief' or 'brief' interventions, which can be used to support MECC. As the name suggests, very brief interventions may only take a few seconds and consist of assessing health status, providing information related to this, and signposting people to where to get help. Brief interventions take a little longer and may involve discussion, encouragement, goal setting, signposting and the provision of supportive materials, for example, information leaflets. Such interventions can be used by any health professional, often without additional training.

Providing brief advice and follow up can lead to health behaviour change, even in people who have not expressed a desire to change (McQueen et al, 2011; Aveyard et al, 2012). A Cochrane review of 49 studies considered nursing or health visitor-delivered interventions for smoking cessation of varying intensity. Overall the likelihood of quitting increased compared with a control or usual care (Rice et al, 2013). However, this was also found in a subgroup analysis of seven studies of a very low intensity intervention, i.e. where brief advice to stop smoking was provided (with or without a leaflet) during a single consultation lasting 10 minutes or fewer with up to one follow-up visit. Similarly, in a systematic review of 13 randomised controlled trials (RCTs) where doctors gave brief, opportunistic advice to patients to stop smoking on medical grounds and signposted to smoking cessation services, this intervention increased the number of quit attempts (risk ratio 1.24, 95\% confidence interval 1.16-1.33) compared with usual care (Aveyard et al, 2012). 
Many of the interventions described above used a variety of evidence-based behaviour change techniques. A selection of those that may be most useful to nurses are described below.

Behaviour change techniques

\section{Very brief advice}

Nurses have many demands on their time. A systematic and structured approach to health promotion can save time and ensure that nurses MECC (Bailey et al, 2012). Smoking is still the most harmful health behaviour found in the general population and the 'Ask, Advise, Act' approach of very brief advice (VBA) was developed as a simple, brief and effective method for busy health professionals to intervene (McEwen and Grothier, 2013). It is a helpful example of how a very brief intervention can be delivered in around 30 seconds and was informed by the Aveyard et al (2012) review (Box 1).

The VBA approach deliberately avoids asking smokers how much they smoke, what they smoke and if they want to quit. This is because asking these questions can make patients feel defensive. Also, unless the nurse asking has been specifically trained in smoking cessation and has the time to enter into a full smoking cessation consultation, it is unlikely to be an effective approach. Online training in the VBA approach, including continuing professional development (CPD) certification, is available without charge from the National Centre for Smoking Cessation and Training:

http://tinyurl.com/pc7c29p.

\section{Goal setting}

Goal setting has been shown to be highly effective in helping people to change behaviour (NICE, 2014). Nurses can help patients to identify a desired goal and then to develop a plan to achieve it. This facilitates small changes that can then lead to long-term improvement (Lorig et al, 2014). It has been proposed that goals accelerate behaviour change through specifying the required actions and subsequently enhancing self-efficacy (Bodenheimer and Handley, 2009; Barley et al, 2014). Achievement of small goals motivates individuals to continue with the new behaviour, or to set a more ambitious goal.

To help patients to set goals that are attainable nurses can use SMART goals. The acronym SMART stands for: specific (exactly what will be achieved), measurable (how to know if it has been achieved), achievable (realistic taking into account skills, resources and context), relevant (important to the person and the outcome they want), time-bound (when the goal will be accomplished). Setting SMART goals has been found to be beneficial for smoking cessation, healthy eating, exercising, oral hygiene, breast self-examination, stair use and taking a vitamin supplement (Kwasnicka et al, 2013). Table 1 is an example of a SMART goal that operationalises an intention to eat more healthily.

\section{Table 1 SMART Goal examples for healthy eating}

General: I must eat more healthily.

Specific: I will eat fruit instead of biscuits between meals. 
Hard to measure: I will eat more fruit and fewer biscuits.

Easy to measure: On weekdays at work I will eat an apple or a banana instead of biscuits. I will remove the biscuits from my desk drawer and leave them in the kitchen for colleagues. I will put a tick in my diary for each day I achieve this. I will aim to do this on 7 of the next 10 working days.

Difficult to achieve: I will only eat healthy food from now on.

Easier to achieve: I will swap biscuits for fruit when at work.

Not relevant: I will eat more healthily because the nurse says I should.

Relevant: I will eat more fruit and fewer biscuits as this will help me lose weight and feel better about myself.

No time frame: I will lose weight.

With time frame: I will achieve my target weight within 6 months.

It is important to write the goal down, under each of the SMART headings, in collaboration with the patient, and to ask patients what they may need to plan in advance to achieve their goal. In the example in Table 1 this may be making a plan to buy fruit on the way to work on a Monday morning.

As with learning any new skill, goals rarely go perfectly to plan from the first attempt, and exploring why the goal was not achieved can inform a more realistic new goal (Michie et al, 2008). Strategies to manage potential barriers and setbacks can be developed and will help prevent 'relapse', where people revert to their old behaviour (Kwasnicka et al, 2013). Nurses may, from their professional experience, be able to predict some likely barriers to achieving health-related goals such as emotional responses, erroneous beliefs or more concrete barriers such as access difficulties or financial concerns. However, asking the patient to identify such barriers ensures that care will be personalised and more meaningful to the patient. Once barriers have been identified, nurses can help the patient to problem solve. Facilitating the patient to come up with their own solutions will increase self-efficacy and the solutions generated are more likely to be achievable. Nurses can prompt patients to find their own solutions by asking questions such as 'what do you think might help?' or 'what has helped you in similar situations?'

\section{Self-monitoring and feedback}

The NICE (2014) guidance on behaviour change identified that using self-monitoring and feedback are more likely to lead to changing health behaviours. Self-monitoring involves individuals keeping track of their own health behaviours' outcomes. For instance, nurses may ask their patients to keep a daily diary record of the number of alcoholic drinks consumed, the number of steps walked, or their lung function. Keeping track provides patients with a much clearer understanding of their current behaviours, what they need to change and when they have been successful, as well as providing vital information for the nurse. It is recommended that self-monitoring is physically recorded in some format, such a writing, keeping track on an app, or even taking photographs.

Feedback is in the form of a review of changes in the behaviour or outcome, which the nurse can carry out with the patient. It is also recommended that patients are encouraged to review the 
changes themselves (or with the help of a carer), to build self-efficacy, independence, and increase self-management skills.

Monitoring and recording behaviour systematically is useful as it provides an objective record of how the patient has behaved, which may differ from their perception of what they have done (Michie et al, 2008). As with many of us, patients are often subject to bias, for instance, as a result of lack of awareness or being reluctant to accept that they are making unhealthy choices. Of course, diary entries may also be biased, for example, because of feelings of shame about a behaviour and not wanting others to know about this. However, the process of systematic self-monitoring increases the individual's awareness of their health behaviours, many of which may have become habitual or automatic. It also helps people to target their behaviour change efforts. For instance, by keeping a daily food diary individuals can clearly see the times of day when they are most at risk of unhealthy eating and can identify which types of food they overindulge in. Reviewing successful progress (feedback) changes behaviour through increasing self-efficacy (Bandura, 1997). Achievements such as healthier eating and increases in physical activity are made explicit and visible, resulting in a virtuous circle of increased confidence that supports and maintains change. When change has been difficult or unsuccessful it can help highlight where the problems are so that these can be addressed, for example through goal setting.

\section{Motivational interviewing}

A more complex intervention for behaviour change that can be used by nurses, but which requires more training, is motivational interviewing (MI) (Miller and Rollnick, 1991). MI is a technique that encourages the patient to reflect on the benefits of change. It is a collaborative approach where, instead of trying to persuade the patient, the nurse uses negotiation skills to help the patient to explore their own motivations to change and the reasons why they may be resisting. A systematic review of Ml analysing 48 RCTs with 9618 participants found it to be effective for a range of outcomes including HIV viral load, dental outcomes, weight, alcohol and tobacco use, and reducing sedentary behaviour (Lundahl et al, 2013). 'More information about MI can be found on the Motivational Interviewing Network of Trainers (MINT) website:

http://www.motivationalinterviewing.org/

\section{Conclusion}

In summary, there is a range of brief, evidence-based behaviour-change techniques that nurses can use to help their patients to make and stick to healthy choices. More details can be found in chapter 4 of Barley (2016).

Health promotion through behaviour change support is a fundamental part of every nurse's role and is key to promoting high-quality care within the NHS. By using evidence-based behaviour-change techniques nurses will be more successful in helping patients to change their behaviour and improve their health.

Key Points

- Providing brief advice and follow up can lead to behaviour change, even in people who have not expressed a desire to change

- Setting SMART goals involves defining a desired behaviour change and the specific actions required to make it happen. Achievement of small goals motivates individuals to continue with the new behaviour or to set more ambitious goals 
- Self-monitoring involves individuals keeping track of their progress. It provides them with a clear understanding of their behaviours, what they need to change and what strategies work

- Nurses can provide feedback that highlights success. This increases patients' self-efficacy, or confidence, to continue with the healthy behaviours

CPD reflective questions

- What kinds of healthy choices would it be beneficial for your patients to make?

- How do you usually encourage patients to make healthy choices? Could you use any of the techniques described here to improve your patient's success?

- What gets in the way of delivering health promotion in your practice? How could these barriers be overcome?

Declaration of interest: none

Further information on this topic can be found in chapter 4 of Barley E (2016) Health Psychology in Nursing Practice. Sage Publications, London

\section{References}

1. Aveyard P, Begh R, Parsons A, West R (2012) Brief opportunistic smoking cessation interventions: a systematic review and meta-analysis to compare advice to quit and offer of assistance. Addiction 107(6): 1066-73. https://dx.doi.org/10.1111/j.1360-0443.2011.03770.x

2. Bailey V, Soni A, Alessi C et al for NHS Future Forum (2012) The NHS's Role in the Public's Health: A Report From the NHS Future Forum. http://tinyurl.com/mskyhk6 (accessed 8 November 2016)

3. Bandura A (1997) Self-Efficacy: The Exercise of Control. Freeman, New York

4. Barley E (2016) Health Psychology in Nursing Practice. Sage, London

5. Barley EA, Walters P, Haddad M et al (2014) The UPBEAT nurse-delivered personalized care intervention for people with coronary heart disease who report current chest pain and depression: a randomised controlled pilot study. PLoS One 9(6):e98704. https://dx.doi.org/10.1371/journal.pone.0098704

6. Bodenheimer T, Handley MA (2009) Goal-setting for behavior change in primary care: an exploration and status report. Patient Educ Couns 6(2):174-80. https://dx.doi.org/10.1016/j.pec.2009.06.001

7. Kwasnicka D, Presseau J, White M, Sniehotta FF (2013) Does planning how to cope with anticipated barriers facilitate health-related behaviour change? A systematic review. Health Psychology Review 7(2):129-45. http://dx.doi.org/10.1080/17437199.2013.766832

8. Lorig K, Laurent DD, Plant K, Krishnan E, Ritter PL (2014) The components of action planning and their associations with behavior and health outcomes. Chronic IIIn 10(1): 50-9. https://dx.doi.org/10.1177/1742395313495572

9. Lundahl B, Moleni T, Burke BL et al (2013) Motivational interviewing in medical care settings: a systematic review and meta-analysis of randomized controlled trials. Patient Educ Couns 93(2):157-68. https://dx.doi.org/10.1016/j.pec.2013.07.012

10. McEwen A, Grothier L (2013) Smoking cessation: the heart of the matter. British Journal of Cardiac Nursing 8(9): 438-42. https://dx.doi.org/10.12968/bjca.2013.8.9.438

11. McQueen J, Howe TE, Allan L, Mains D, Hardy V (2011) Brief interventions for heavy alcohol users admitted to general hospital wards. Cochrane Database Syst Rev (8): CD005191. https://dx.doi.org/10.1002/14651858.CD005191.pub3 
12. Michie S, Rumsey N, Fussell A et al for British Psychological Society Division of Health Psychology Team (2008) Improving Health: Changing Behaviour. NHS Health Trainer Handbook. Department of Health, London

13. Miller WR, Rollnick S (1991) Motivational Interviewing: Preparing People to Change Addictive Behaviour. 1st edn. Guilford Press, New York

14. National Institute for Health and Care Excellence (2014) Behaviour change: individual approaches. [PH49]. https://www.nice.org.uk/guidance/ph49 (accessed 8 November 2016)

15. Rice VH, Hartmann-Boyce J, Stead LF (2013) Nursing interventions for smoking cessation. Cochrane Database Syst Rev (8): CD001188 